\title{
On the asymptotic solutions of singulary perturbed differ- ential systems of fractional order
}

\author{
Burkhan Kalimbetov $^{\mathrm{a}, *}$, Elmyra Abylkasymova ${ }^{\mathrm{b}}$, Gulbakhram Beissenova ${ }^{\mathrm{b}, \mathrm{c}}$ \\ ${ }^{a}$ Khoja Ahmet Yasawi International Kazakh-Turkish University, B. Sattarkhanov 29, Turkestan, Kazakhstan. \\ ${ }^{b}$ University of Friendship of People's Academican A. Kuatbekov, Toleby 32, Shymkent, Kazakhstan. \\ ${ }^{c}$ M. Auezov South Kazakhstan University, Take-Khan avenue 7, Shymkent, Kazakhstann.
}

\begin{abstract}
In this paper, we consider an initial problem for systems of differential equations of fractional order with a small parameter for the derivative. A regularization problem is produced, and an algorithm for normal and unique solubility of general iterative systems of differential equations with partial derivatives is given. In the environment of the computer mathematical system Maple, approximate solutions are calculated, and corresponding solution schedules for various values of the small parameter are constructed.
\end{abstract}

Keywords: Matrix-function, vector-function, differential equation of fractional order, regularization, asymptotic, iterative problems, normal and unique solvability.

2020 MSC: 34A08, 34E10, 34E15.

(C)2022 All rights reserved.

\section{Introduction}

We consider the following singularly perturbed problem:

$$
L_{\varepsilon} y(t, \varepsilon) \equiv \varepsilon y^{(1 / 3)}-A(t) y=h(t), \quad y(0, \varepsilon)=y^{0}, \quad t \in[0, T],
$$

where $y(t, \varepsilon) \equiv\left\{y_{1}, \ldots, y_{n}\right\}$ is unknown vector-function, $h(t) \equiv\left\{h_{1}, \ldots, h_{n}\right\}$ is a known vector-function, $A(t)-n \times n$ is a matrix-function, $y^{0}=\left\{y_{1}^{0}, \ldots, y_{n}^{0}\right\}$ is a known constant vector, and $\varepsilon>0$ is a small parameter. It is required to construct a regularized asymptotic of a solution $[5,6,11]$ of the problem $(1.1)$ at for $\varepsilon \rightarrow+0$.

Problem (1.1) is a Cauchy problem for an ordinary differential equation of fractional order. According to the definition of a fractional order derivative [7-9], i.e., $y^{(\alpha)}(t)=t^{(1-\alpha)} y^{\prime}(t), 0<\alpha<1$, where $y^{\prime}(t)$ is derivative of the first order from the function $y(t)$ by the variable $t$, we write the problem (1.1) in the following form:

$$
L_{\varepsilon} y(t, \varepsilon) \equiv \varepsilon \sqrt[3]{t^{2}} \frac{d y}{d t}-A(t) y=h(t), \quad y(0, \varepsilon)=y^{0}, \quad t \in[0, T]
$$

\footnotetext{
${ }^{*}$ Corresponding author

Email address: burkhan.kalimbetov@ayu.edu.kz (Burkhan Kalimbetov)
}

doi: $10.22436 /$ jmcs.024.02.07

Received: 2020-02-20 Revised: 2020-12-13 Accepted: 2021-01-01 
It should be noted that singularly perturbed differential equations with fractional derivatives from the standpoint of the regularization method [11], from the perspective of the regularization method were considered in [2-4]. We will consider the problem (1.2) under the following assumptions:

1) matrix-function $A(t)$ and vector-function $h(t)$ belong to the space $C^{\infty}[0, T]$, that is elements of the matrix-function $A(t)$ and components of the vector $h(t)$ have derivatives of any order on the segment $[0, \mathrm{~T}]$

2) the matrix-function $A(t)$ has the Jordan structure, such that roots of the characteristic equation

$$
\operatorname{det}[A(t)-\lambda I]=0,
$$

where $I$ is unit matrix of the $n \times n$, order, satisfy the following requirements:

a) $\lambda_{i}(t) \neq \lambda_{j}(t), \quad i \neq j, \quad \lambda_{j}(t) \neq 0, \quad j=\overline{1, n}$;

b) $\operatorname{Re} \lambda_{j}(t) \leqslant 0, j=1, n, \quad \forall t \in[0, T]$.

\section{Regularization of the problem (1.2)}

We introduce regularizing variables [11]:

$$
\tau_{j}=\frac{1}{\varepsilon} \int_{0}^{t} s^{(-1 / 3)} \lambda_{j}(s) d s \equiv \varphi_{j}(t, \varepsilon), j=\overline{1, n},
$$

and instead of the problem (1.2), we will consider "extended" problem

$$
L_{\varepsilon} \tilde{y}(t, \tau, \varepsilon) \equiv \varepsilon \sqrt[3]{t^{2}} \frac{\partial \tilde{y}}{\partial t}+\sum_{j=1}^{n} \lambda_{j}(t) \frac{\partial \tilde{y}}{\partial \tau_{j}}-A(t) \tilde{y}=h(t), \tilde{y}(0,0, \varepsilon)=y^{0} .
$$

Relation of the problem (2.1) with the problem (1.2) is that if $\tilde{y}(t, \tau, \varepsilon)$ is a solution of the problem (2.1), then contraction of the solution $\tilde{y}\left(t, \varphi_{j}(t, \varepsilon), \varepsilon\right) \equiv y(t, \varepsilon)$ when $\tau_{j}=\varphi_{j}(t, \varepsilon), j=\overline{1, n}$ will be exact solution of the problem (1.2).

Defining a solution of the system (2.1) in the form of series:

$$
\tilde{y}(t, \tau, \varepsilon)=\sum_{k=0}^{\infty} \varepsilon^{k} y_{k}(t, \tau), y_{k}(t, \tau) \in C^{\infty}\left([0, T], C^{n}\right),
$$

we obtain the following iteration problems:

$$
\begin{aligned}
\operatorname{L} y_{0}(t, \tau) & \equiv \sum_{j=1}^{n} \lambda_{j}(t) \frac{\partial y_{0}}{\partial \tau_{j}}-A(t) y_{0}=h(t), y_{0}(0,0)=y^{0}, \\
L_{y_{1}}(t, \tau) & =-\sqrt[3]{t^{2}} \frac{\partial y_{0}}{\partial t}, \quad y_{1}(0,0)=0, \\
& \vdots \\
L y_{k}(t, \tau) & =-\sqrt[3]{t^{2}} \frac{\partial y_{k-1}}{\partial t}, \quad y_{k}(0,0)=0, \quad k \geqslant 1 .
\end{aligned}
$$

\section{Solvability of iteration problems}

Solution of each of the iteration problems (2.2)-(2.4) will be defined in the space $U$ of functions of the form:

$$
u=\left\{y(t, u): y=y_{0}(t)+\sum_{j=1}^{n} y_{j}(t) e^{\tau_{j}}, \quad y_{j}(t) \in C^{\infty}\left([0, T], C^{n}\right)\right\}
$$


Each of the iteration problems (2.2)-(2.4) has the following form:

$$
L y(t, \varepsilon) \equiv \sum_{j=1}^{n} \lambda_{j}(t) \frac{\partial y_{0}}{\partial \tau_{j}}-A(t) y_{0}=h(t, \tau),
$$

where $h(t, \tau) \in U$ is corresponding right hand side.

The following Theorem takes place.

Theorem 3.1. Let $\mathrm{h}(\mathrm{t}, \tau) \in \mathrm{U}$ and conditions 1) and 2a) hold. Then, for solvability of the equation (3.2) in space $\mathrm{U}$, it is necessary and sufficient that the following conditions hold:

$$
<\mathrm{h}(\mathrm{t}, \tau), \mathrm{d}_{\mathfrak{j}}(\mathrm{t})>\equiv 0, \quad \mathrm{j}=\overline{1, \mathrm{n}}, \quad \forall \mathrm{t} \in[0, \mathrm{~T}],
$$

where $d_{j}(t)$ are eigenfunctions of the matrix of functions $A^{*}(t)$, corresponding to eigenvalues $\bar{\lambda}_{j}(t), j=\overline{1, n}$.

Proof. Defining a solution $y(t, \tau)$ of the system (3.2) as an element (3.1) of the space $\mathrm{U}$, we get the following systems for the coefficients $y_{j}(t), j=\overline{0, n}$, of the sum (3.1):

$$
\begin{aligned}
{\left[\lambda_{k}(t) I-A(t)\right] y_{k}(t) } & =h_{k}(t), \quad k=\overline{1, n}, \\
-A(t) y_{0}(t) & =h_{0}(t), \quad(I \equiv \operatorname{diag}(1,1)) .
\end{aligned}
$$

The system (3.5), due to $\operatorname{det} A(t) \neq 0$, has a unique solution $y_{0}(t)=-A^{-1}(t) h_{0}(t)$. The system (3.4) is solvable in $C^{\infty}[0, T]$ if and only if the condition $<h_{k}(t), d_{k}(t)>\equiv 0, k=\overline{1, n}, \forall t \in[0, T]$, holds, that coincides with the condition (3.3). Theorem 3.1 is proved.

Remark 3.2. If the conditions (3.3) hold, system (3.2) has a solution that can be represented as

$$
y(t, \tau)=\sum_{k=1}^{n}\left[\alpha_{k}(t) c_{k}(t)+\sum_{\substack{s \neq k \\ s=1}}^{n} \frac{\left(h_{k}(t), d_{s}(t)\right)}{\lambda_{k}(t)-\lambda_{s}(t)} c_{s}(t)\right] e^{\tau_{k}}-A^{-1}(t) h_{0}(t),
$$

where $\alpha_{k}(t) \in C^{\infty}[0, T], k=\overline{1, n}$ are arbitrary scalar functions.

The following theorem establishes conditions under which the solution (3.6) of system (3.2) is uniquely defined in the class $\mathrm{U}$.

Theorem 3.3. Let 1) and 2a) hold and $h(t, \tau) \in U$ of the system (3.2) satisfies conditions (3.3). Then the system (3.2) with additional conditions:

$$
\begin{gathered}
y(0,0)=y^{0}, \\
<-\sqrt[3]{t^{2}} \frac{\partial y(t, \tau)}{\partial t}, d_{j}(t)>\equiv 0, \quad j=\overline{1, n}, \quad \forall t \in[0, T],
\end{gathered}
$$

where $\mathrm{y}^{0} \in \mathrm{C}^{\mathrm{n}}$ are known constants, is uniquely solvable in the space $\mathrm{U}$.

Proof. Since conditions of Theorem 3.1 hold, the system (3.2) has a solution in the space $U$ in the form (3.6), where functions $\alpha_{k}(t), k=\overline{1, n}$ have not yet been found. To calculate them, we will use additional conditions (3.7) and (3.8).

We subject (3.7) to the initial condition (3.8), we get the system:

$$
\sum_{k=1}^{n}\left[\alpha_{k}(0) c_{k}(0)+\sum_{s \neq k, s=1}^{n} \frac{\left(h_{k}(0), d_{s}(0)\right)}{\lambda_{k}(0)-\lambda_{s}(0)} c_{s}(0)\right]-A^{-1}(0) h_{0}(0)=y^{0} .
$$


Multiplying scalarly both sides of this equality by $d_{k}(0)$ and taking into account biorthogonality of the systems $\left\{c_{k}(t)\right\}$ and $\left\{d_{k}(0)\right\}$, we uniquely find initial values $\alpha_{k}(0)=\alpha_{k}^{0}$ for the functions $\alpha_{k}(t), k=\overline{1, n}$.

We subject now the function (3.6) to the condition (3.8). First calculate $\frac{\partial y(t, \tau)}{\partial t}$ :

$$
\sum_{k=1}^{n}\left\{\left(\alpha_{k} c^{\prime}{ }_{k}+\alpha^{\prime}{ }_{k} c_{k}\right)+\left[\sum_{s \neq k, s=1}^{n} \frac{\left(h_{k}, d_{s}\right)^{\prime}\left(\lambda_{k}-\lambda_{s}\right)-\left(h_{k}, d_{s}\right)\left(\lambda_{k}-\lambda_{s}\right)^{\prime}}{\lambda_{k}-\lambda_{s}} c_{s}+\frac{\left(h_{k}, d_{s}\right)}{\lambda_{k}-\lambda_{s}} c^{\prime}{ }_{s}\right]\right\} e^{\tau_{k}}-\left(A^{-1} \cdot h_{0}\right)^{\prime} .
$$

Conditions (3.8) lead to the equations:

$$
-\sqrt[3]{t^{2}}\left[\alpha^{\prime}{ }_{k}+\left(c^{\prime}{ }_{k}, d_{k}\right) \alpha_{k}+\sum_{\substack{s \neq k \\ s=1}}^{n} \frac{\left(h_{k}, d_{s}\right)}{\lambda_{k}-\lambda_{s}}\left(c^{\prime}{ }_{k}, d_{k}\right)-\left(\left(A^{-1} \cdot h_{0}\right)^{\prime}, d_{k}\right)\right]=0, \quad k=\overline{1, n},
$$

which together with the initial conditions $\alpha_{k}(0)=\alpha_{k}^{0}$, found earlier, allow us to uniquely find the functions $\alpha_{k}(t), k=\overline{1, n}$. Theorem 3.3 is proved.

\section{Exsamples}

Example 4.1. Using the algorithm developed above, construct the main term of the asymptotic solution of the Cauchy problem:

$$
\varepsilon\left(\begin{array}{l}
y^{(1 / 3} \\
z^{(1 / 3)}
\end{array}\right)=\left(\begin{array}{cc}
0 & 1 \\
-1 & 0
\end{array}\right)\left(\begin{array}{l}
y \\
z
\end{array}\right)+\left(\begin{array}{l}
h_{1}(t) \\
h_{2}(t)
\end{array}\right), \quad \begin{aligned}
& y(0, \varepsilon)=y^{0} \\
& z(0, \varepsilon)=z^{0}
\end{aligned}
$$

where $t \in[0, T], T<1, \varepsilon>0$ small parameter. Eigenvalues of the matrix-function $A(t)$ of this system are numbers $\lambda_{1}(t) \equiv-i, \lambda_{2}(t) \equiv+i$. The corresponding eigenvectors $c_{\mathfrak{j}}(t)$ and eigenvectors $d_{j}(t)$ of the conjugate operator $A^{*}(t)$ have the form:

$$
c_{1}=\left(\begin{array}{c}
-i \\
-1
\end{array}\right), c_{2}=\left(\begin{array}{c}
i \\
-1
\end{array}\right), d_{1}=\left(\begin{array}{l}
i \\
1
\end{array}\right), d_{2}=\left(\begin{array}{c}
-i \\
1
\end{array}\right)
$$

Introduce regularizing variables:

$$
\tau_{1}=-\frac{3 i}{2 \varepsilon} \sqrt[3]{t^{2}} \equiv \varphi_{1}(t, \varepsilon), \quad \tau_{2}=\frac{3 i}{2 \varepsilon} \sqrt[3]{t^{2}} \equiv \varphi_{2}(t, \varepsilon)
$$

For extended functions $\tilde{u} \equiv\{\tilde{y}(t, \tau, \varepsilon), \tilde{z}(t, \tau, \varepsilon)\}$ we obtain the following problem:

$$
\varepsilon \sqrt[3]{t^{2}} \frac{\partial \tilde{u}}{\partial t}+\sum_{j=1}^{2} \lambda_{j} \frac{\partial \tilde{u}}{\partial \tau_{j}}-A \tilde{u}=h(t), \quad \tilde{u}(0,0, \varepsilon)=u^{0},
$$

where $\tilde{u}=\{\tilde{y}, \tilde{z}\}, h(t)=\left\{h_{1}(t), h_{2}(t)\right\}, u^{0}=\left\{y^{0}, z^{0}\right\}$. Defining a solution of this problem in the form of series

$$
\tilde{u}(t, u, \varepsilon)=\sum_{k=0}^{\infty} \varepsilon^{k} u_{k}(t, u),
$$

we get the following iteration systems:

$$
\mathrm{L}_{0} \mathrm{u}_{0}(\mathrm{t}, \tau) \equiv \sum_{j=1}^{2} \lambda_{j} \frac{\partial \mathrm{u}_{0}}{\partial \tau_{j}}-A u_{0}=h(t), \quad u_{0}(0,0)=u^{0}
$$




$$
\begin{array}{rlrl}
\mathrm{L}_{0} \mathrm{u}_{1}(\mathrm{t}, \tau) & =-\sqrt[3]{\mathrm{t}^{2}} \frac{\partial \mathrm{u}_{0}}{\partial t}, & \mathfrak{u}_{1}(0,0)=0, \\
\mathrm{~L}_{0} \mathrm{u}_{\mathrm{k}}(\mathrm{t}, \tau)=-\sqrt[3]{\mathrm{t}^{2}} \frac{\partial \mathrm{u}_{k-1}}{\partial t}, & \mathfrak{u}_{\mathrm{k}}(0,0)=0, \quad k \geqslant 1 .
\end{array}
$$

We look for a solution of the equation (4.2) in the form of the functions:

$$
u_{0}(t, \tau)=u_{1}^{(0)}(t) e^{\tau_{1}}+u_{2}^{(0)}(t) e^{\tau_{2}}+u_{0}^{(0)}(t)
$$

Putting (4.4) into the equation (4.2), and equating coefficients at the same exponentials and the free terms, we get:

$$
\begin{aligned}
{\left[\lambda_{1} I-A\right] u_{1}^{(0)}(t) } & =0, \\
{\left[\lambda_{2} I-A\right] u_{2}^{(0)}(t) } & =0, \\
-A u_{0}^{(0)}(t) & =h(t) .
\end{aligned}
$$

From the system (4.7) we find

$$
u_{0}^{(0)}(t)=-A^{-1} h(t)
$$

In the equations $(4.5)$ and $(4.6), u_{1}^{(0)}(t), u_{2}^{(0)}(t)$ are arbitrary functions. Thus, we have defined solution (4.4) of the system (4.2) in the following way:

$$
u_{0}(t, \tau)=\alpha_{1}^{(0)}(t) c_{1} e^{\tau_{1}}+\alpha_{2}^{(0)}(t) c_{2} e^{\tau_{2}}-A^{-1} h(t),
$$

where $\alpha_{k}^{(0)}(t), k=1,2$, are arbitrary functions. We subject $(4.8)$ to the initial condition $u_{0}(0,0)=u^{0}$.

$$
\left(\begin{array}{l}
y^{0} \\
z^{0}
\end{array}\right)=\alpha_{1}^{(0)}(0)\left(\begin{array}{l}
-i \\
-1
\end{array}\right)+\alpha_{2}^{(0)}(0)\left(\begin{array}{c}
i \\
-1
\end{array}\right)-\left(\begin{array}{cc}
0 & -1 \\
1 & 0
\end{array}\right)\left(\begin{array}{l}
h_{1}(0) \\
h_{2}(0)
\end{array}\right)
$$

or

$$
\left\{\begin{array}{c}
-i \alpha_{1}^{(0)}(0)+i \alpha_{2}^{(0)}(0)+h_{2}(0)=y^{0} \\
-\alpha_{1}^{(0)}(0)-\alpha_{2}^{(0)}(0)-h_{1}(0)=z^{0}
\end{array}\right.
$$

then we get:

$$
\alpha_{1}^{(0)}(0)=\frac{z^{0}-h_{1}(0)-i\left[h_{2}(0)-y^{0}\right]}{2}, \quad \alpha_{2}^{(0)}(0)=\frac{z^{0}+h_{1}(0)+i\left[h_{2}(0)-y^{0}\right]}{2} .
$$

To uniquely define arbitrary functions $\alpha_{k}^{(0)}(t), k=1,2$, that are present in the solution (4.8) of the problem (4.2), we proceed to the next iteration problem (4.3). First we calculate:

$$
\frac{\partial u_{0}(t, \tau)}{\partial t}=\dot{\alpha}_{1}^{(0)}(t) c_{1} e^{\tau_{1}}+\dot{\alpha}_{2}^{(0)}(t) c_{2} e^{\tau_{2}}-A^{-1} \dot{h}(t) .
$$

Solution of the equation (4.3) is sought as a function:

$$
w_{1}(t, \tau)=w_{1}^{(1)}(t) e^{\tau_{1}}+w_{2}^{(1)}(t) e^{\tau_{2}}+w_{0}^{(1)}(t) .
$$

Substituting (4.11) into the equation (4.3) (taking into account (4.10)), and equating coefficients at the same exponentials and the free terms, we have:

$$
\left[\lambda_{1} I-A\right] u_{1}^{(1)}(t)=-\sqrt[3]{t} \dot{\alpha}_{1}^{(0)}(t), \quad\left[\lambda_{2} I-A\right] u_{2}^{(1)}(t)=-\sqrt[3]{t} \dot{\alpha}_{2}^{(0)}(t), \quad-A u_{0}^{(1)}(t)=-\sqrt[3]{t} A^{-1} \dot{h}(t) .
$$


For solvability of the first two systems it is necessary and sufficient that

$$
\dot{\alpha}_{k}^{(0)}(t)=0, \quad k=1,2 .
$$

Taking into account the initial conditions (4.9), we find the functions

$$
\alpha_{1}^{(0)}(\mathrm{t})=\alpha_{1}^{(0)}(0) \equiv \frac{z^{0}-h_{1}(0)-\mathfrak{i}\left[h_{2}(0)-y^{0}\right]}{2}, \quad \alpha_{2}^{(0)}(\mathrm{t})=\alpha_{2}^{(0)}(0) \equiv \frac{z^{0}+h_{1}(0)+i\left[h_{2}(0)-y^{0}\right]}{2},
$$

unambiguously. Thus, we defined arbitrary functions $\alpha_{k}^{(0)}(t)=0, k=1,2$, in the solution (4.8), and thereby, uniquely determined the function (4.4) of the iteration problem (4.2), i.e., built the main term of the asymptotics of solutions to the problem (4.1):

$$
\begin{aligned}
\left(\begin{array}{c}
y_{\varepsilon 0}(t) \\
z_{\varepsilon 0}(t)
\end{array}\right)= & {\left[\frac{z^{0}-h_{1}(0)-i\left(h_{2}(0)-y^{0}\right)}{2}\right]\left(\begin{array}{c}
-i \\
-1
\end{array}\right) e^{-\frac{3 i}{2 \varepsilon} \sqrt[3]{t^{2}}} } \\
& +\left[\frac{z^{0}+h_{1}(0)+i\left(h_{2}(0)-y^{0}\right)}{2}\right]\left(\begin{array}{c}
i \\
-1
\end{array}\right) e^{\frac{3 i}{2 \varepsilon} \sqrt[3]{t^{2}}}-\left(\begin{array}{cc}
0 & -1 \\
1 & 0
\end{array}\right)\left(\begin{array}{c}
h_{1}(t) \\
h_{2}(t)
\end{array}\right) .
\end{aligned}
$$

Example 4.2. Find approximate solutions and build graphs of the system solution

$$
\varepsilon\left(\begin{array}{c}
y^{(1 / 3)}(t, \varepsilon) \\
z^{(1 / 3)}(t, \varepsilon)
\end{array}\right)=\left(\begin{array}{cc}
0 & 1 \\
-1 & 0
\end{array}\right)\left(\begin{array}{l}
y(t, \varepsilon) \\
z(t, \varepsilon)
\end{array}\right)+\left(\begin{array}{c}
e^{t} \\
-e^{t}
\end{array}\right), \quad y(0,1)=1, \quad z(0,1)=1,
$$

for different values $\varepsilon$ in the environment of the computer mathematical system Maple $[1,10]$.

$>$ restart; cond: $=y(0.1)=1, z(0.1)=1$;

$$
\text { cond }:=y(0.1)=1, z(0.1)=1 ;
$$

$\left.\left.>\operatorname{sys} 1:=\operatorname{diff}\left(0.1^{*} y(t), t\right)=(z(t)+\exp (t)) /(t \hat{1} 1 / 3)\right),\left(\operatorname{diff}\left(0.1^{*} z(t), t\right)=(-y(t)-\exp (t)) /(t \hat{(} 1 / 3)\right)\right) ;$

$$
\text { sys } 1:=0.1\left(\frac{d}{d t} y(t)\right)=\frac{z(t)+e^{t}}{t^{(1 / 3)}}, 0.1\left(\frac{d}{d z} z(t)\right)=\frac{-y(t)-e^{t}}{t^{(1 / 3)}}
$$

$>$ dsolve $(\operatorname{sys} 1, y(t), z(t))$

$$
\begin{aligned}
\{y(t)= & \sin \left(15 t^{(2 / 3)}\right) \_C 2+\cos \left(15 t^{(2 / 3)}\right) \_C 1+\int \frac{\cos \left(15 t^{(2 / 3)}\right) e^{t}\left(t^{(1 / 3)}-10\right)}{t^{(1 / 3)}} d t \sin \left(15 t^{(2 / 3)}\right) \\
& -\int \frac{\sin \left(15 t^{(2 / 3)}\right) e^{t}\left(t^{(1 / 3)}-10\right)}{t^{(1 / 3)}} d t \cos \left(15 t^{(2 / 3)}\right), \\
z(t)= & \cos \left(15 t^{(2 / 3)}\right) \int \frac{\cos \left(15 t^{(2 / 3)}\right) e^{t}\left(t^{(1 / 3)}-10\right)}{t^{(1 / 3)}} d t \\
= & \left.\sin \left(15 t^{(2 / 3)}\right) \int \frac{\sin \left(15 t^{(2 / 3)}\right) e^{t}\left(t^{(1 / 3)}-10\right)}{t^{(1 / 3)}} d t+\cos \left(15 t^{(2 / 3)}\right) \_C 2-\sin \left(15 t^{(2 / 3)}\right) \_C 1-e^{t}\right\}
\end{aligned}
$$

$$
\begin{aligned}
& \text { sys2 }:=0.2\left(\frac{d}{d t} y(t)\right)=\frac{z(t)+e^{t}}{t^{(1 / 3)}}, 0.2\left(\frac{d}{d t} z(t)\right)=\frac{-y(t)-e^{t}}{t^{(1 / 3)}} ; \\
& \text { sys3 }:=0.5\left(\frac{d}{d t} y(t)\right)=\frac{z(t)+e^{t}}{t^{(1 / 3)}}, 0.5\left(\frac{d}{d t} z(t)\right)=\frac{-y(t)-e^{t}}{t^{(1 / 3)}} ; \\
& \text { sys4 }:=0.65\left(\frac{d}{d t} y(t)\right)=\frac{z(t)+e^{t}}{t^{(1 / 3)}}, 0.65\left(\frac{d}{d t} z(t)\right)=\frac{-y(t)-e^{t}}{t^{(1 / 3)}} ;
\end{aligned}
$$



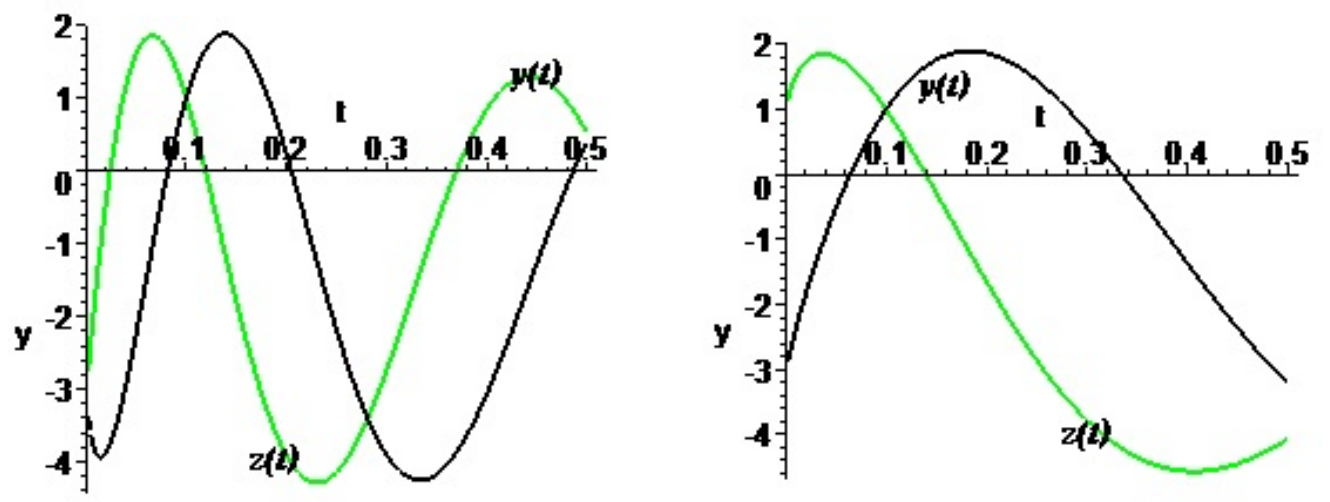

Figure 1: epsilon=0.1, epsilon=0.2.

$$
\begin{aligned}
& \text { sys5 }:=0.08\left(\frac{d}{d t} y(t)\right)=\frac{z(t)+e^{t}}{t^{(1 / 3)}}, 0.08\left(\frac{d}{d t} z(t)\right)=\frac{-y(t)-e^{t}}{t^{(1 / 3)}} ; \\
& \text { sys6 }:=0.05\left(\frac{d}{d t} y(t)\right)=\frac{z(t)+e^{t}}{t^{(1 / 3)}}, 0.05\left(\frac{d}{d t} z(t)\right)=\frac{-y(t)-e^{t}}{t^{(1 / 3)}} ; \\
& \text { sys7 }:=0.01\left(\frac{d}{d t} y(t)\right)=\frac{z(t)+e^{t}}{t^{(1 / 3)}}, 0.01\left(\frac{d}{d t} z(t)\right)=\frac{-y(t)-e^{t}}{t^{(1 / 3)}} ; \\
& \text { sys8 }:=0.005\left(\frac{d}{d t} y(t)\right)=\frac{z(t)+e^{t}}{t^{(1 / 3)}}, 0.005\left(\frac{d}{d t} z(t)\right)=\frac{-y(t)-e^{t}}{t^{(1 / 3)}} .
\end{aligned}
$$
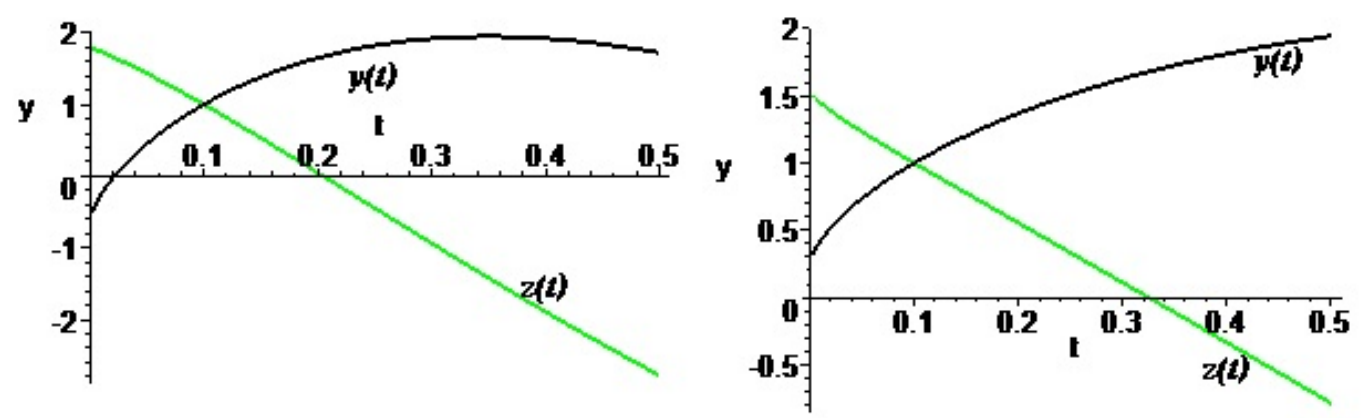

Figure 2: epsilon=0.5, epsilon=0.65.
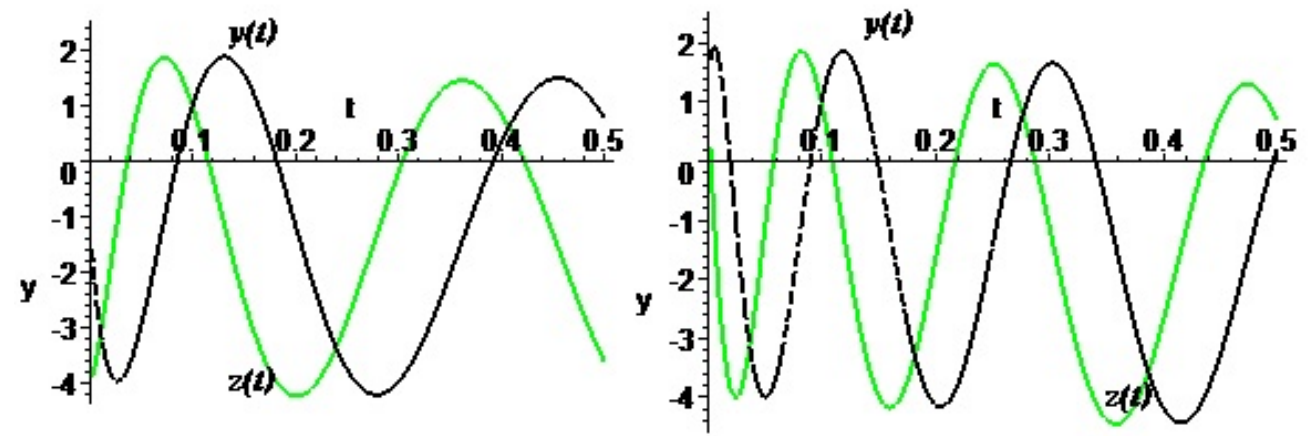

Figure 3: epsilon=0.08, epsilon=0.05. 

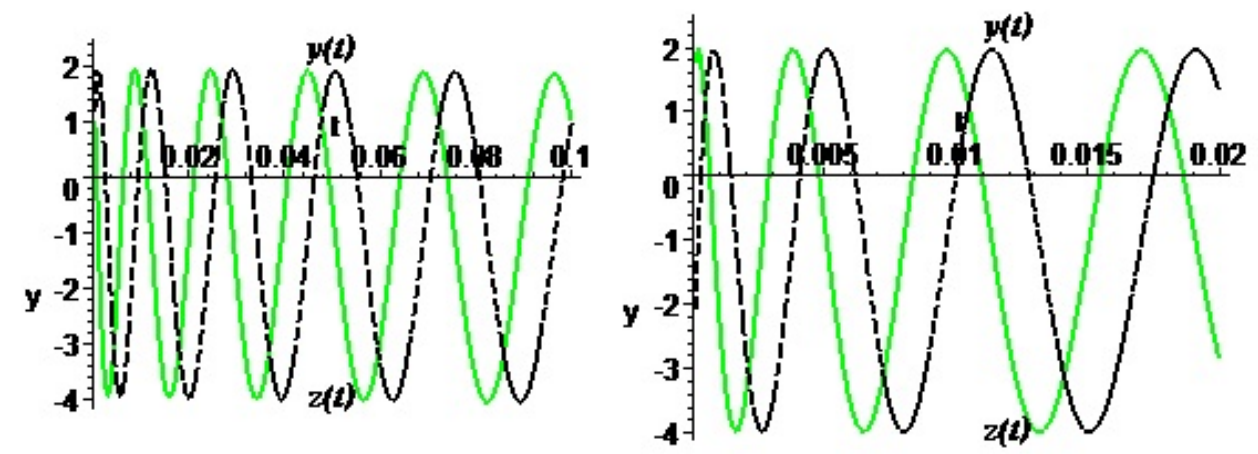

Figure 4: epsilon=0.01, epsilon=0.005.

\section{Acknowledgment}

This work is supported by the grant AP05133858 "Contrast structures in singularly perturbed equations and their application in the theory of phase transitions" by the Scientific Committee of the Ministry of Education and Science of the Republic of Kazakhstan.

\section{References}

[1] V. P. Dyakonov, Maple 7. Training course, Peter, St. Petersburg, (2003). 4.2

[2] B. T. Kalimbetov, On the question of asymptotic integration of singularly perturbed fractional order problems, Asian J. Fuzzy Appl. Math., 6 (2018), 44-49. 1

[3] B. T. Kalimbetov, Regularized asymptotics of solutions for systems of singularly perturbed differential equations of fractional order, Int. J. Fuzzy Math. Arch., 16 (2018), 67-74.

[4] B. T. Kalimbetov, Scalar singularly perturbed Cauchy problem for a differential equation of fractional order, Asian J. Fuzzy Appl. Math., 7 (2019), 10-13. 1

[5] B. T. Kalimbetov, V. F. Safonov, A regularization method for systems with unstable spectral value of the kernel of the integral operator, J. Differ. Equ., 31 (1995), 647-656. 1

[6] B. T. Kalimbetov, M. A. Temirbekov, Z. O. Khabibullayev, Asymptotic solutions of singular perturbed problems with an instable spectrum of the limiting operator, Abstr. Appl. Anal., 2012 (2012), 16 pages. 1

[7] U. N. Katugampola, Correction to "What is a fractional derivative?" by Ortigueira and Machado, J. Comput. Phys., 321 (2016), 1255-1257. 1

[8] R. Khalil, M. Al Horani, D. Anderson, Undetermined coefficients for local fractional differential equations, J. Math. Comput. Sci., 16 (2016), 140-146.

[9] R. Khalil, M. Al Horani, A. Yousef, M. Sababheh, A new definition of fractional derivative, Comput. Appl. Math., 264 (2014), 65-70. 1

[10] M. N. Kirsanov, Graphs in Maple, Fizmatlit, Moscow, (2007). 4.2

[11] S. A. Lomov, Introduction to General Theory of Singular Perturbations, American Mathematical Society, Providence, (1992). 1, 1, 2 